\title{
SPECIAL CARE AFTER THORACOTOMY
}

\author{
By E. F. ChIN, M.B., B.S., F.R.C.S. \\ Surgeon in charge, Thoracic Unit, Southampton Chest Hospital
}

It is only logical that some remarks on preoperative care be submitted in a paper which is dealing with post-operative care of cases who have undergone thoracotomy, as certain aspects follow through directly and have an effect on the postoperative sequel. Some features are no more important to thoracic work than to general surgical work, such as care and attention to the patient's dental hygiene, general nutrition and the blood picture. There are specific features in relation to thoracic work and these are as follows:

(I) Pulmonary Infections. These must be controlled pre-operatively. There are those cases coming to thoracotomy who have chronic lowgrade infection, seen usually in the older age groups who suffer from so-called 'bronchitis.' These patients are prone to pulmonary infections and often have associated spasm. Another group are those with severe pulmonary suppuration, and, indeed, it might be for this suppuration that they are coming to surgery, such cases being bronchiectasis and chronic lung abscess.

It will depend upon the degree of infection what treatment is undertaken. It is remarkable what improvement can be made by simple means such as tipping and breathing exercises and activity. These patients should be encouraged to be as active as possible and not to lie inertly in their beds all day, but to be up, fully dressed and about, to take exercise outside the wards, and if such facilities as a gymnasium are available, to use that, particularly in competitive games. If suppuration persists, and the patient is still producing mucopus which is not diminished after adequate tipping and exercise, then a course of antibiotics is well worth while just prior to surgery; which drug is used will depend upon the organisms and their sensitivities. In certain pulmonary cases the preoperative preparation might take some weeks, and this is particularly seen in children with bronchiectasis.

(2) Breathing Exercises. Breathing exercises, to be effective, must be carried out diligently under the instruction of a physiotherapist who really understands broncho-pulmonary anatomy and the pathology with which she is dealing. It is useless to have an overworked physiotherapist visiting the patient for five minutes once a day, as so often happens. Also it is desirable that the patients should fully understand what the breathing exercises are for, and should do their exercises in classes to engender a competitive spirit. The breathing exercises should be made very simple and it is most desirable to reduce them to the simplest common factor, so that in the post- $\vec{N}$

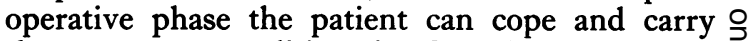
them out as a conditioned reflex. The system of external respiration should be completely ignored and concentration made entirely on perfecting diaphragmatic movement and control. The
reasons for this are:

(a) Man, being an orthograde animal, is diaphragmatic breather. If fully trained in simp o diaphragmatic movement, he then is not cauglo up in the post-operative period between the systern of internal respiration and external respiration. Diaphragmatic movement is effective and is particularly effective for lifting the bronchial secretions.

(b) Diaphragmatic breathing is painless after $\stackrel{\mathbb{2}}{2}$ thoracotomy and the patient who has a large $\overrightarrow{\overrightarrow{0}}$ thoracotomy scar with a tube in the pleural space $\frac{3}{3}$

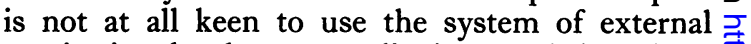
respiration, but he can use diaphragmatic breathing painlessly.

It must be stressed that the habit of diaphragmatic breathing is so established in the patient $\frac{\sigma}{3}$ that he does it automatically before he comes to surgery and with the slightest encouragement in the immediate post-operative phase uses his diaphragm entirely.

(3) Activity. Pre-operative activity should be $D$ stressed. In some cases, of course, this is neither possible nor desirable. For example, cases in cardiac failure need rest, not activity. But in the main patients should be up and about, fully $\mathbb{N}$ dressed, and not slopping about in slippers and $\omega$ dressing-gowns. They should be encouraged to take walks and to get out of the atmosphere of theco ward. This improves their general health and improves their muscle tone.

(4) $X$-ray. An X-ray should always be taken $\frac{7}{\circ}$ on admission and a further X-ray should be taken $\overrightarrow{\mathbb{D}}$ 
the day before operation. The importance of the pre-operative X-ray cannot be over-stressed. Of course, there is no point in taking an $\mathrm{X}$-ray if it is not viewed.

(5) Nose Swab. On admission all cases should have a nose swab taken, and the only organism of interest is that of the staph. aureus, particularly phage type 8o. The reason for this is to stop the ward becoming endemic of this organism and to treat this patient so that he does not come to the theatre with a heavy growth of staph. aureus in his nose, which well may end up in a postoperative staph. aureus pneumonia. The best way of treating such a case is by a spray consisting of tyrothricin ( 0.05 per cent. in $40 \mathrm{ml}$.), neomycin $(280 \mathrm{mg}$.) and aqua ad. (100 $\mathrm{ml}$.). The nose should be sprayed with this two-hourly. After two or three days' treatment further swabs are taken and treatment continued until negative cultures are obtained.

(6) Patients over the age of 50 should be digitalized. A small percentage of cases in the older age group, but still a significant percentage, tend to fibrillate after a thoracotomy. It is desirable to digitalize these patients pre-operatively, so that if fibrillation does occur they are under control and it causes little disability. Digitalis can be stopped 10 to 12 days post-operatively if sinus rhythm has been maintained.

The role of the pre-operative phase is to improve the general health of the patient and to increase his activity; in other words, to train him for the operation. Of course, this is not always possible, but it is desirable in those cases in which it can be managed. An effort should be made to improve the respiratory function, to clear the bronchial tree of infection and to train the patient in such habits as effective coughing, diaphragmatic breathing, and to explain to the patient why these manoeuvres are being undertaken and the fact that he is being trained in these habits purely for the post-operative phase. In other words, the patient should be in the know.

\section{Post-operative Period}

This can be divided into three phases:

\section{(a) In the Theatre}

(i) Blood Replacement. This should be complete and it is a good idea to know what the blood loss has been. With very little trouble this can easily be measured by the weight of the swabs and the measurement of blood in aspiration bottles. It can be taken that I gm. = I c.c. of blood. Swab weighing in lots of 10 is the best method and, while the estimate of $\mathrm{I}$ gm. = I c.c. is not quite accurate, it is adequate to use as a basis for blood replacement.

(ii) It is unnecessary to perform a rib resection at thoracotomy. The only time this is necessary is when doing a thoracoplasty. There is no other thoracic operation which requires rib resection. The best approach is through the periosteal bed of the rib required. After this has been effected the back end of the rib should be divided and $\frac{1}{2}$ in. resected; this prevents apposition of the ends and thus lessens pain. The reasons for avoiding a rib resection are that it is completely unnecessary and it makes for a much more painful post-operative course. There is little doubt that patients who have had the intercostal nerve removed have a much less painful post-operative course than those in whom it remains, and hence it is desirable that the intercostal nerve be taken proximal to the rib division.

(iii) Before the endo-bronchial tube is removed, complete suction should be carried out via the tube, and if this is found not to be complete, then the patient should be bronchoscoped.

(iv) The patient, before leaving the theatre, should be breathing on his own and coughing at request. This should be the routine and not the exception.

(v) With few exceptions, all thoracotomies should have drainage on to an under-water seal. The tube should be of adequate size which will drain fluid and air easily. The tube is inserted through a stab incision in the lower intercostal space. This incision should be made long enough, so that the tube is not causing pressure on the skin, or else it will be painful. In those cases of pulmonary resection the tube, while inserted basally, is brought up to the apex. This siting of the tube makes it much more efficient for the drainage of air and fluid. A tube in this position rarely becomes blocked. Any residual fluid at the base can easily be obtained by tipping the end of the bed, laying the patient flat and putting gentle suction through the under-water system. Oesophageal cases have a basal tube which is quite adequate.

(vi) Pneumonectomies are an exception and drainage is unnecessary. If the patient is operated on in the lateral position, when he is turned on to his bed the intrapleural pressure must be stabilized to atmospheric, and if a Maxwell box is not avaik able do this with a wide-bore needle inserted through an intercostal space, preferably the second anteriorly. This will stabilize the pleural pressures to atmospheric if it is left in while the patient takes several breaths.

(vii) The chest should not be closed until the lung is seen to be fully expanded.

\section{(b) In the Ward-Immediate}

The patient should have a half-hourly blood pressure recording and the drip is discontinued when the pulse rate and the blood pressure readings 
are satisfactory. When these readings are satisfactory the patient can be slowly sat up. There are exceptions to this routine, but very few. In cardiac cases it might be desirable to leave the patient lying supine longer than in pulmonary or oesophageal cases. In all cardiac cases the pulse rate alone is not adequate, as this will not reveal whether there is a pulse deficit present or not, and a chart should be kept of the apex beat as well as the pulse rate.

While most cases are nursed in a semi-recumbent or sitting position, there are exceptions to this. In lobectomies and segmental resections it is a good practice to nurse the patient on his side with the good side down. The advantages of this are that there is good bronchial drainage from the operated side and the costo-phrenic angle is kept free of fluid, so that there cannot be any obliteration of the costo-phrenic sulcus and thus subsequent impairment of function on the operated side.

The relief of pain is important. The patient who has pain cannot cough and cannot co-operate. His pain should be relieved forthwith. There is no contraindication to the use of morphine derivatives. Coughing is an active spontaneous action and nothing will kill the patient's spontaneity more than pain. It is essential for the nurse or the physiotherapist to realize when the patient has bronchial secretions which need to be coughed up and to try to get the patient to indulge in vigorous coughing. The patient must be aided by having his chest firmly supported (Fig. I). However, when the patient is suffering intense pain the first duty of the doctor or the nurse is to relieve the pain and then to encourage the patient in his expectoration.

The under-water drainage should be checked and it should be seen that it is swinging and that it is not blocked. In the early post-operative course the commonest cause of block is by the patient lying on it. It is also important that the underwater drainage bottle be kept in a tin with sand in the bottom, so that it cannot be kicked over. A bottle being kicked over and broken in the night can cause a patient's death by a total pneumothorax.

In this immediate phase the patient should be visited by the physiotherapists, and in a unit which is carrying out thoracotomies this should be as much a routine as the recording of the patient's blood pressure. Sometimes a patient becomes panicky, but with a hand on the abdomen the patient will steady down and control his respiration, and this is where the importance of good pre-operative training pays its dividends. If secretions are present, the patient should be encouraged to cough and he should cough until he has lifted his secretions. The only important aspect of this post-operative period is to make sure the patient is not retaining bronchial secretions, and both the nurses and the physiotherapists should be्ष on the constant look-out for this and encourage thea patient to clear his bronchial tree of anyz obstruction.

Full arm movements should be gone throughe within two hours. There is little doubt that if $a \stackrel{\Rightarrow}{\Rightarrow}$ full range of movement is gone through within two hours, and is repeated several times in the next 24 을 hours, there will be no trouble. But it is much more difficult to get a patient to go through full $\frac{\bar{c}}{{ }^{*}}$ arm movements if this initial action is delayed for $\cong$ 24 hours.

\section{(c) Intermediate Period}

During the whole of the post-operative period $\vec{\omega}$ the patient should clear himself of any bronchials secretions and this is not a manoeuvre whicho should be indulged in just in the immediate period 3 . and then forgotten about. It should be persisted $\omega_{\omega}^{\omega}$ with until the patient leaves the hospital. It is $\omega_{0}$ desirable to remove the under-water drainage as quickly as possible, as this renders the patient? immobile and it is often uncomfortable and often ${ }^{N}$ painful. Most cases should have the tube removed? on the following day, but this is not always possible, $\vec{\nabla}$ particularly after segmental resections, where theree may be an air leak which persists for some days. If after segmental resection or lobectomy air leak뭉 does persist, it is better to change the big basal tube after three days and insert a small apical tuk $\overrightarrow{0}$ as most drainage will have ceased by now and the tube is merely there to remove air. The whote. object of post-operative thoracic care is to close the pleural space. Of course, this is quite impossible after a pneumonectomy, as there is nothing to close the pleural space with, but with this one $\frac{}{\alpha}$ exception the pleural space should be closed. $A \stackrel{\Omega}{\rightarrow}$ closed pleural space is a safe pleural space, and an $\overrightarrow{\overrightarrow{0}}$ open space is a potential danger. The factors which 3 keep a pleural space open are: (i) atelectasis, (ii) air in the pleura, and (iii) sero-sanguinous effusion. These will be discussed in detail. In a pneu- $\frac{0}{3}$ monectomy aspiration may be necessary if mediastinal shift is occurring. This can readily be seen 3 . on the X-ray, but is often apparent clinically, as aid deviation of the trachea to the contralateral side is $\frac{3}{3}$ apparent and the patient may be complaining of $\frac{\mathrm{o}}{\mathrm{S}}$ increasing dyspnoea.

(i) Atelectasis. Prevention is better than cure, and $\frac{7}{2}$ the rate of atelectasis following a thoracotomy is a direct reflection on the standard of physiotherapyos and nursing, but even with the highest standards $N$ atelectasis will still occur intermittently. Atelectasis N may not always be recognized clinically by the ${ }_{\sigma}^{\omega}$ doctor, the physiotherapist or the nurses, but it should be picked up on the X-ray, and that is why it is essential that all these cases have daily X-rays. Frequently it should be picked up by the house ${ }_{\tau}^{+}$ 


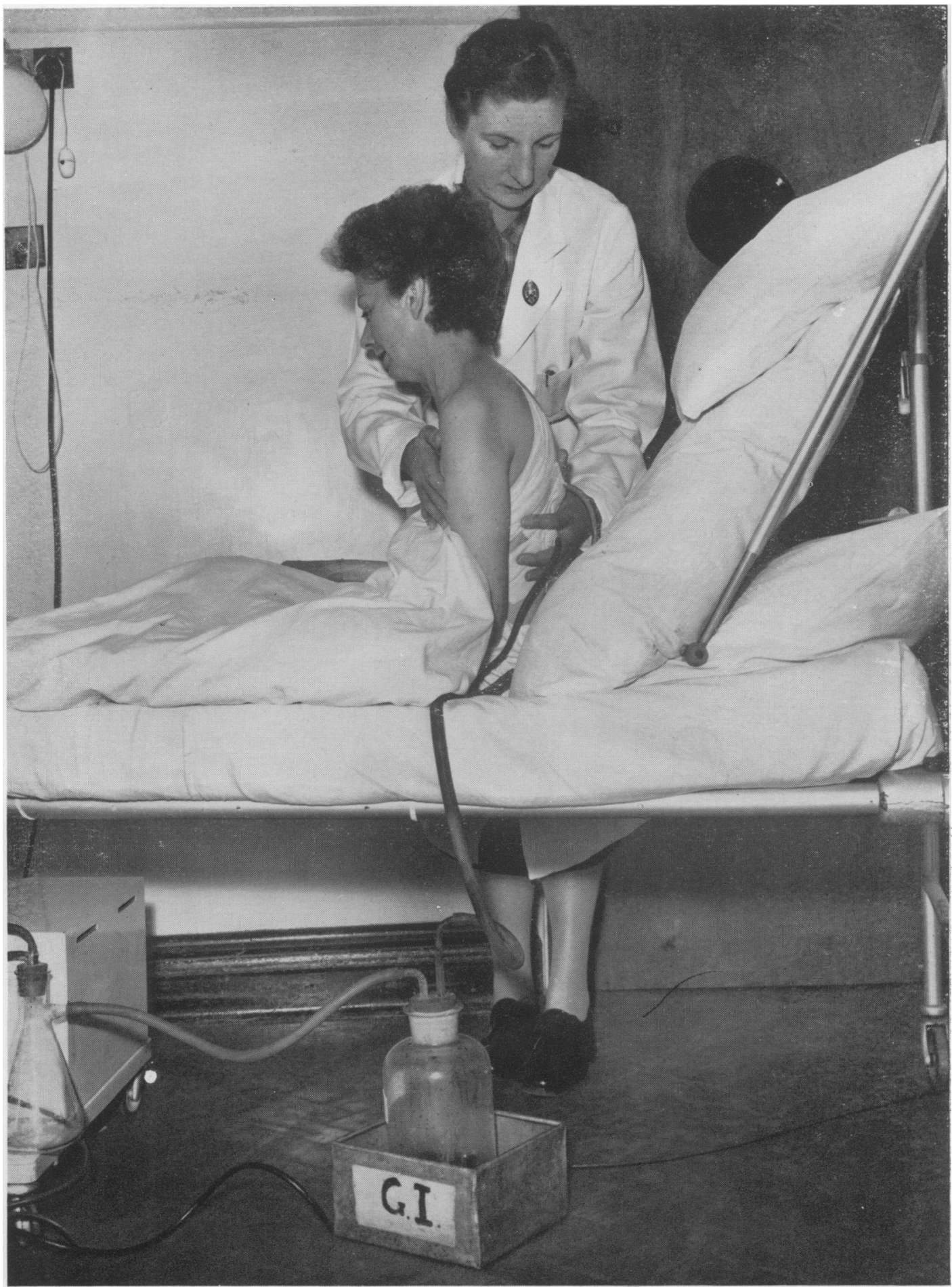

Fig. 1.-The photograph illustrates a patient in bed being coughed after decortication. Effective coughing cannot be carried out unless the operated side is supported firmly, as is shown in the photograph. The coughing, to be effective, must be done in short bursts so that the patient is not tired easily. 


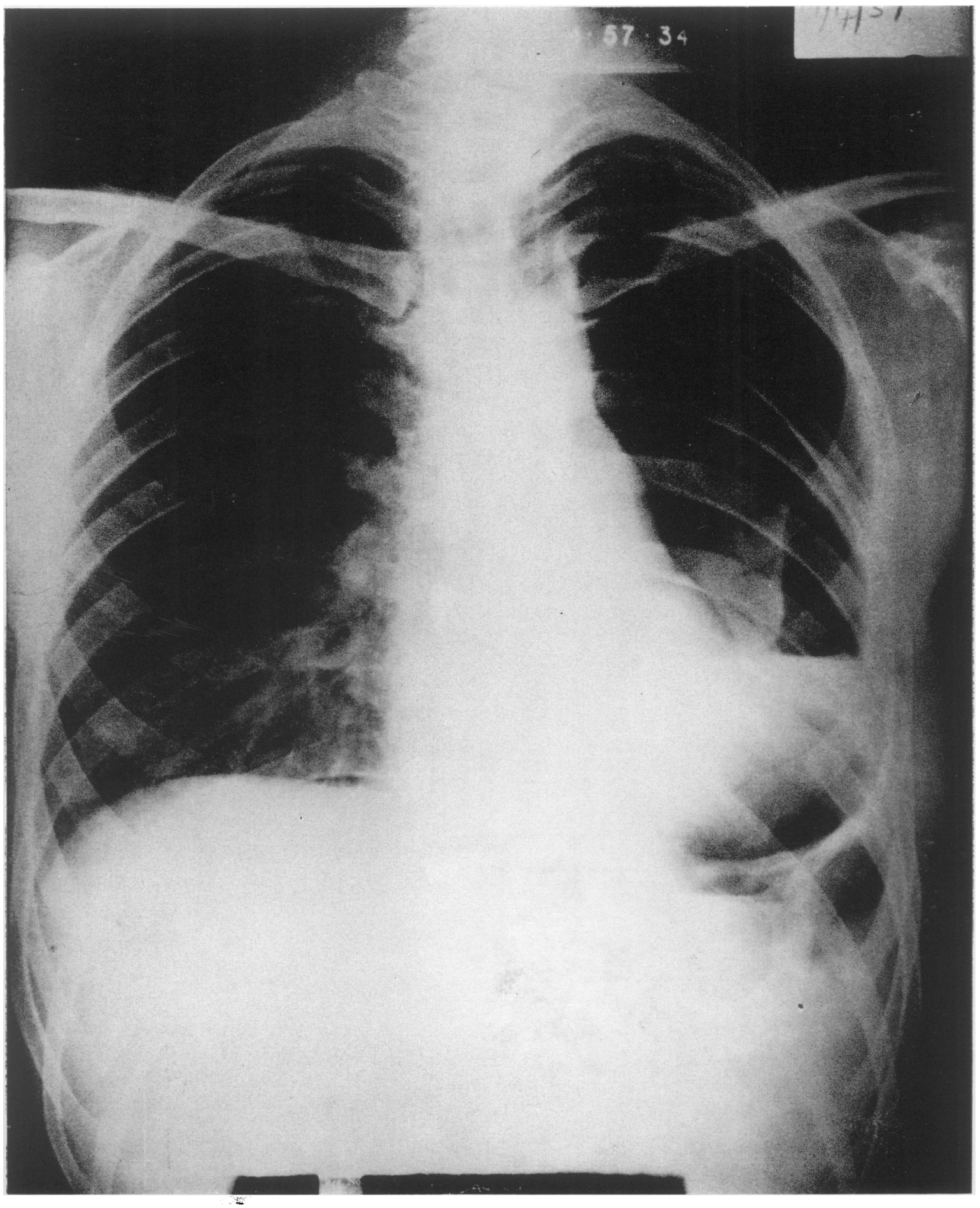

Fig. 2. - Shows a post-operative $\mathrm{X}$-ray where everything is wrong. There is a hydro-pneumothorax and some atelectasis. This patient was handled by being bronchoscoped first of all and then the air and fluid were removed by aspiration. It is important that bronchoscopy be carried out prior to aspiration, as the lung cannot be expanded if there is a central block, and if the pleura is aspirated with a central block the high negative pressure obtained tends to impact the mucus plug further. 
staff or the physiotherapists or nurses. There is no place for laissez-faire and letting atelectasis look after itself. It must be treated energetically. The immediate handling should be conservative, in that the patient is given an inhalation and is put into the hands of the physiotherapists, when the patient is coughed vigorously and tipped. If this fails to relieve the bronchial obstruction, then the patient must be bronchoscoped. There is no need to bring the patient to the theatre to do this. It is quite a simple matter to bronchoscope the patient sitting up in bed. Once the atelectasis is eradicated there is a tendency for it to recur owing to oedema of the bronchial mucosa, and coughing should be continued and the patient given bronchodilators. The whole manoeuvre may have to be repeated subsequently and once the vicious circle of recurring atelectasis is set up it may be difficult to break, because it results in pulmonary infection, an increase in the pulmonary secretions and an increase in the oedema of the bronchial mucosa.

(ii) Air in the Pleura. If the post-operative $\mathrm{X}$-ray shows a pneumothorax, then the underwater drainage should be connected to continuous suction and a Roberts suction pump is a very adequate form of continuous suction. Suction must be continued until there is no escape or air. Once this has ceased, then the pump can be discontinued and the tube removed only after it has been left in for a further 12 hours and X-ray shows that there is no re-accumulation of air.

(iii) Sero-sanguinous Effusion. The daily postoperative $\mathrm{X}$-rays will show whether there is any effusion present or not and any effusion should be aspirated. The best means of aspiration is by a Martin's syringe, which has a three-way tap with bayonet fittings and will allow no air to enter the chest. The chest should be aspirated to dryness.

It will be seen from the above paragraphs that it is essential that these patients should be X-rayed daily post-operatively until the pleural space is completely clear, and then they should have check $\mathrm{X}$-rays every two or three days until they are ready for discharge. All patients are got up as quickly as possible, and even if it is found necessary to retain their intercostal tube, it is still desirable to get them out of bed into a chair. In the immediate post-operative phase a rocking chair is the best sort of chair, as it allows the patient to exercise his limbs and thus cuts down the incidence of pulmonary embolism. Patients are encouraged to get fully dressed once their tubes are out, and to walk around. The fact that they are walking about and dressed does not mean that they cannot get postoperative complications and they still should be watched very carefully and the daily $\mathrm{X}$-ray should be checked for atelectasis and effusion.

To summarize, basically the essence of postoperative care in thoracotomy cases is to obliterate the pleural space, and this applies to segmental resection, lobectomy, oesophagectomy, repair of hiatal hernia, vagotomy, sympathectomy, cardiac operation, and, indeed, any operation which demands a thoracotomy. While many operations present peculiar problems of their own (for example, oesophagectomy demands the handling of other features, such as the fluid balance of the patient and a careful watch for paralytic ileus, and cardiac operations demand careful watch for arrhythmias and cardiac failure), a common factor in all these is to prevent pulmonary atelectasis, to prevent the accumulation of air and fluid in the pleural space and to obliterate the pleural space, as an obliterated pleural space is a safe pleural space. Fig. 2 shows every undesirable feature. Combined with atelectasis there exists a hydropneumothorax. In such a case bronchoscopy should be performed and after the bronchus block has been removed then the air and fluid completely aspirated.

\section{RUTHIN CASTLE, NORTH WALES}

A Clinic for the diagnosis and treatment of Internal Diseases (except Mental or Infectious Diseases). The Clinic is provided with a staff of doctors, technicians and nurses.

The surroundings are beautiful. The climate is mild. There is central heating throughout. The annual rainfall is 30.5 inches, that is less than the average for England.

The Fees are inclusive and vary according to the room occupied.

For particulars apply to THE SECRETARY, Ruthin Castle, North Wales.

Telegrams: Castle, Ruthin

Telephone: Ruthin 66 